\title{
A Comparison of Transmission Schemes for Scalable Video over MIMO Relay Networks
}

\author{
Phuc Chau*, Yongwoo Lee*, Jitae Shin* \\ *School of Electronic and Electrical Engineering, Sungkyunkwan University, Suwon, Rep. of Korea \\ cmphuc@skku.edu, tencio2001@skku.edu,jtshin@skku.edu
}

\begin{abstract}
This paper investigates a comparison of transmission schemes for the scalable video over multiple-input multipleoutput relay networks. We consider three time-division multipleaccess cooperative schemes by using embedded space time codes with various degrees of broadcasting and receiving collision. In this paper, we assume that base station and relay station are equipped two antennas and mobile stations have only one antenna. The relay station operates decode-and-forward mode for cooperative transmission. The base station broadcasts scalable video bit stream by encoding embedded space time codes with the assistance of relay. Upon the amount of received information of each cooperative scheme, Mobile stations get the corresponding efficient decoding. With the help of relayed information, base layer retrieves better protection for ensuring the basic quality of scalable video. Visual quality is refined by enhancement layers carried in transmitted signal. We investigate the diversity performance of various schemes in term of bit error rate. Scalable video performance is also provided for comparing and evaluating the effectiveness of scalable video transmission schemes. Based on the need of users, it's possible to select the suitable scheme for trading off between the protection of base and enhancement layers.
\end{abstract}

Keywords — Fading relay channels, scalable video coding, spacetime coding, broadcast channel.

\section{INTRODUCTION}

The significant progresses of both wireless data communication and video compression technique have enabled digital wireless broadcast application such as digital video broadcast (DVB) and high-definition video on demand (HDVOD). On one hand, advanced multiple-input multipleoutput (MIMO) wireless physical-layer technique has significantly increased the spatial diversity and spatial multiplexing [1]. On the other hand, the scalable video coding extension of the H.264/Advanced Video Coding (SVC) standard provides high coding efficiency and scalability. The scalable video content is split into scalable layers to provide the different quality of services (QoS). The base layer (BL) guarantees the basic quality of supported video performance, whereas the enhancement layers (ELs) provide the refinement information of the aforementioned base layer [2].

In designing scalable video transmission system, it is important to highly protect the BL for guaranteeing the basic video quality. Because the bit streams of different layers have different importance. Unequal error protection (UEP) and forward error correcting protection (FEC) are usually applied for the system according to the source significant information [3], [4].

Most mobile stations (MSs) have limited size and resource. Therefore, cooperative communications have been proposed in many works not only to exploit the spatial diversity without the need of multiple antennas at each one but also to combat or reduce the effect of fading without additional power or any sacrifice in bandwidth [5]-[8]. Three time-division multipleaccess (TDMA) cooperative protocols were analysed and examined in [9]. The authors considered a simple fading relay channels where source, relay and destination are each equipped with single antenna transceiver. They provided the comparison of the aforementioned three TDMA protocols by using mutual information and outage capacity analysis.

Space time coding (STC) was applied to increase the spatial diversity with the famous one Alamouti's code proposed in 1998 [10]. C.-H. Kuo proposed embedded space time codes (E-STC) for broadcasting and cooperative transmission broadcast using E-STC, respectively [11], [12]. In [11], the ESTC was proposed for wireless multimedia broadcast with heterogeneous receivers. Receivers with low configuration decode only base layer information while the receivers with more antennas can retrieve more information of transmitted layers. To the best of our knowledge, the paper [12] was the first one which considered cooperative transmission using ESTC for scalable video broadcast system. The base station (BS) is equipped two antennas and each user has only one antenna transceiver. Multiple layers are broadcasted by BS, on the other end link, MSs with one antenna is able to decode $\mathrm{BL}$ information for experiencing the basis video quality. For cooperative transmission case, terminal with the help from other terminals working as relay terminal can decode more layers and get the full quality of supported video by provider. The authors use amplify-and-forward (AF) mode for relay terminal in cooperative communication. The cooperative transmission consists of two time slots. In the first time slot, the BS broadcasts multiple layers to relay station (RS) and MSs. The RS amplifies and forwards the received signal from BS to MSs in the second time slot. Because both the scalable layer information and noise are amplified at RS, so MSs can't get good decoding if the source-relay channel is not sufficiently reliable. 
In this paper, we apply E-STC for different fading relay channels over MIMO relay networks. We make a comparison of various transmission schemes for scalable video. Our work is related to the papers [9]-[12]. In MIMO relay networks where two antennas are equipped at BS and RS, only one antenna at MSs to account for size and resource constraint. The RS operates decode and forward (DF) mode. Upon more independently received signal, the MSs get better decoding efficiency with the higher video layer. Our main contributions are summarized as follows.

- We establish a unified framework for the combination of E-STC and fading relay channels over MIMO relay networks. Three possible transmission schemes are compared for video wireless broadcast system. The MSs get benefits from fading relay channels for giving more protection with a guarantee of the BL decoding. They, moreover, retrieve EL for enjoying the full quality of SVC bit stream.

- Both layers are orthogonal space time block codes (OSTBC). Consequently, we use the Alamouti's code decoding technique standard for decoding enhancement layers provides lower complexity than minimum meansquare error (MMSE) equalizer which was used in the papers [11].

- The bit error rate (BER) analysis and scalable video performance are respectively used to investigate the spatial diversity and the effectiveness of the three possible transmission schemes in term of peak signal to noise ratio (PSNR).

- Based on the need of users and comparison result, it's possible to select the suitable transmission scheme.

The rest of this paper is organized as follows. Section II describes transmission schemes over MIMO relay networks. Section III provides BER performance and the related scalable video performance analyses. We conclude in Section IV.

For notation in this paper, the bold lower case letter indicates a vector and the bold upper case letter represents a matrix. Superscript $(\cdot)^{*}$ denotes complex conjugate for scalar and element-wise complex conjugate for matrix. Matrix element $a_{j, i}$ denotes the entity in the $j^{\text {th }}$ row and $i^{\text {th }}$ column of the matrix A. $C N\left(\mu, \sigma^{2}\right)$ represents the circular symmetric complex Gaussian random variable with mean $\mu$ and variance $\sigma^{2}$.

\section{Transmission SCHEMES OVER MIMO RELAY NETWORKS}

\section{A. Transmission Systems}

The fading relay channel of a video broadcast system is depicted in Figure 1. We assume that the propagation environment is flat fading Rayleigh channel in which the channel coefficient remains unchanged during the transmission of single space time block code. The channel state information (CSI) is estimated perfectly at receivers. The BS encodes the media source into OSTBC and broadcasts to $\mathrm{MS}_{\mathrm{k}}$ with the assistance of the RS terminal. The $\mathrm{MS}_{\mathrm{k}}$ denotes the $k^{\text {th }}$ mobile station. Suppose that SVC video is encoded/decoded by JSVM software [13]. The video bit stream is partitioned into two layers with unequal importance: a BL and an EL. The both layers are sent to BS for broadcasting through independent paths from two antennas by using quadrature phase shift keying (QPSK) modulation and E-STC. The goal of cooperative communication is the guarantee of BL transmission. In DF mode, the received signal from BS is demodulated and decoded before retransmission. The RS decodes only the BL from received signal instead of all scalable layers for increasing the protection of the BL.

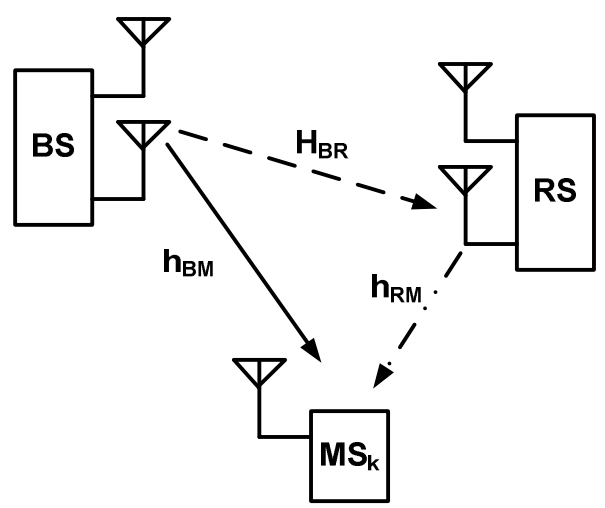

Figure 1. Fading relay channel of a video broadcast system.

Three possible transmission schemes used for scalable video over MIMO relay networks are summarized in Table I.

Scheme I. The BS broadcasts both layers in the first time slot to RS and MSs. In the second time slot, BS sends both layers and the RS sends decoded BL to MSs at the same time. MSs receive the superposition of two signals.

Scheme II. The BS communicates to RS and MSs at the first time slot with both layers. In the second time slot, only RS communicates to MSs.

Scheme III. The BS communicates to RS and MSs in the first time slot with only BL. In the second time slot, BS broadcasts both layers and the RS transmits BL to MSs at the same time.

TABLE 1. ThrEe DIFFERENT TRANSMISSION SCHEMES

\begin{tabular}{|c|c|c|c|}
\hline $\begin{array}{c}\text { Scheme } \\
\text { Time Slot }\end{array}$ & I & II & III \\
\hline 1 & $\begin{array}{c}\text { BS } \rightarrow \text { RS, MSs } \\
\text { (both layers) }\end{array}$ & $\begin{array}{c}\text { BS } \rightarrow \text { RS, MSs } \\
\text { (both layers) }\end{array}$ & $\begin{array}{c}\text { BS } \rightarrow \text { RS, MSs } \\
\text { (only BL) }\end{array}$ \\
\hline 2 & $\begin{array}{c}\text { BS } \rightarrow \text { MSs } \\
\text { (both layers) } \\
\text { RS } \rightarrow \text { MSs (BL) }\end{array}$ & $\begin{array}{c}\text { RS } \rightarrow \text { MSs } \\
\text { (BL) }\end{array}$ & $\begin{array}{c}\text { BS } \rightarrow \text { MSs } \\
\text { (both layer), } \\
\text { RS } \rightarrow \text { MSs (BL) }\end{array}$ \\
\hline
\end{tabular}

\section{B. Transmitter Design}

Suppose that the transmit power at RS is equal to the transmit power at BS which is determined according to the available CSI at RS. The BS sends a code block within two consecutive time slots represented by

$$
\mathbf{S}=\left[\begin{array}{ll}
S_{11} & S_{12} \\
S_{21} & S_{22}
\end{array}\right]
$$

where $s_{i t}$ is the modulated symbol from $i^{\text {th }}$ antenna at time slot $t$. 
We modify the E-STC developed in [12] so that the BS transmits two layer sources. We use orthogonal Alamouti's code for both layers instead of only first layer in [12]. In each code block, the first layer carries modulated symbols $x_{1}$ and $x_{2}$, and second layer conveys modulate symbols $y_{1}$ and $y_{2}$. The transmission matrix of the first layer $\mathbf{C}_{1}$ is represented as

$$
\mathbf{C}_{\mathbf{1}}=\left[\begin{array}{cc}
x_{1} & -x_{2}^{*} \\
x_{2} & x_{1}^{*}
\end{array}\right]
$$

And the second layer code block $\mathbf{C}_{2}$ is

$$
\mathbf{C}_{2}=\left[\begin{array}{cc}
y_{1} & -y_{2}^{*} \\
y_{2} & y_{1}^{*}
\end{array}\right]
$$

Because the most important layer is base layer, $\mathbf{C}_{\mathbf{1}}$ is assigned higher transmit power. Supposing that $\mathbf{C}_{\mathbf{1}}$ and $\mathbf{C}_{\mathbf{2}}$ use same QPSK modulation technique. The transmitted symbol is the superposition of both layers.

$$
\mathbf{S}=\mathbf{C}_{1}+\rho \mathbf{C}_{2}
$$

where $\rho<1$ is amplitude ratio of second layer to first layer.

Absolutely, the interference of both layers is able to occur and could be dealt with orthogonal space time code. The goal of the proposed system is to provide better scalable layers transmission. The quality and performance between both layers could be traded off by adjusting the signal power $\rho$.

\section{Receiver Design}

First we consider non-cooperative case, the $\mathrm{MS}_{\mathrm{k}}$ receives signal directly from BS expressed by

$$
\boldsymbol{r}_{b}=\sqrt{\frac{P_{b}}{2}} \boldsymbol{h}_{B M} \mathbf{S}+\boldsymbol{n}_{B M}
$$

where $P_{b}$ is BS power, $\boldsymbol{h}_{B M}$ denotes channel vector, $\boldsymbol{n}_{B M} \sim C N(0,1)$ is additive white Gaussian noise (AWGN) with zero mean and variance one.

$\mathrm{MS}_{\mathrm{k}}$ only receives one signal comprising both layers from BS. The standard Alamouti decoding principle is available to recover the base layer $\mathbf{C}_{\mathbf{1}}$.

$$
\begin{aligned}
& \widehat{x_{b 1}}=\mu\left[h_{B M 1}^{*} r_{b 1}+h_{B M 2} r_{b 2}^{*}\right] \\
& \widehat{x_{b 2}}=\mu\left[h_{B M 2}^{*} r_{b 2}+h_{B M 1} r_{b 1}^{*}\right]
\end{aligned}
$$

where $\widehat{x_{b j}}$ is the $j^{\text {th }}$ estimated symbol of BL, $\mu=$ $1 /\left(\left|h_{B M 1}\right|^{2}+\left|h_{B M 2}\right|^{2}\right)$ is the normalization factor, $h_{B M j}$ denotes channel coefficient at $j^{\text {th }}$ antenna, $r_{b j}$ indicates the received signal at $j^{\text {th }}$ time slot.

To decode EL, we subtract out the term with $\mathbf{C}_{\mathbf{1}}$ in $\boldsymbol{r}_{b}$ of (5)

$$
\Delta \boldsymbol{r}_{b}=\boldsymbol{r}_{b}-\boldsymbol{h}_{B M} \widehat{\boldsymbol{C}_{1}}
$$

where $\Delta \boldsymbol{r}_{b}$ denotes the residual vector of the remaining layer consisting of noise. The standard Alamouti decoding principle is also used to decode enhancement layer $\mathbf{C}_{\mathbf{2}}$.

Next we consider fading relay channels in order to achieve spatial diversity with the assistance of the RS.

Scheme I. The signal matrix at the RS and the signal vector received at $\mathrm{MS}_{\mathrm{k}}$ in the first time slot are given as, respectively

$$
\begin{gathered}
\boldsymbol{R}_{r}=\sqrt{\frac{P_{b}}{2}} \boldsymbol{H}_{B R} \mathbf{S}+\boldsymbol{N}_{B R} \\
\boldsymbol{r}_{b, 1}=\sqrt{\frac{P_{b}}{2}} \boldsymbol{h}_{B M} \mathbf{S}+\boldsymbol{n}_{B M}
\end{gathered}
$$

where $\boldsymbol{r}_{b, j}$ is the received signal at $j^{\text {th }}$ time slot, $\boldsymbol{n}_{B R} \sim C N(0,1)$ and $\boldsymbol{n}_{B M} \sim C N(0,1)$ are additive white Gaussian noise (AWGN). The RS receives the signal from BS and uses the available standard decoding technique for decoding data before retransmission. In the second time slot, BS and RS communicate to $\mathrm{MS}_{\mathrm{k}}$ at the same time. The received signal is the superposition of two signals.

$$
\boldsymbol{r}_{b, 2}=\sqrt{\frac{P_{b}}{2}} \boldsymbol{h}_{B M} \mathbf{S}+\sqrt{\frac{P_{r}}{2}} \boldsymbol{h}_{R M} \widehat{\mathbf{C}_{\mathbf{1}}}+\widetilde{\boldsymbol{n}}
$$

where the effective noise term $\widetilde{\boldsymbol{n}}=\boldsymbol{n}_{B M}+\boldsymbol{n}_{R M}, P_{r}=P_{b}$ is transmit power at RS.

$\mathrm{MS}_{\mathrm{k}}$ receives two signals within two time slots of (9), (10), respectively. These two signals are sent to the maximum likelihood detector for decoding $\mathrm{BL}$.

$$
\begin{aligned}
& \widehat{x_{b 1}}=\mu\left[\begin{array}{c}
h_{B M 11}^{*} r_{b 1,1}+h_{B M 21} r_{b 2,1}^{*} \\
+\left(h_{B M 12}^{*}+h_{R M 1}^{*}\right) r_{b 1,2}+\left(h_{B M 22}+h_{R M 2}\right) r_{b 2,2}^{*}
\end{array}\right] \\
& \widehat{x_{b 2}^{*}}=\mu\left[\begin{array}{c}
h_{B M 21} r_{b 2,1}+h_{B M 11} r_{b 1,1}^{*} \\
+\left(h_{B M 22}^{*}+h_{R M 2}^{*}\right) r_{b 2,2}+\left(h_{B M 12}+h_{R M 1}\right) r_{b 1,2}^{*}
\end{array}\right]
\end{aligned}
$$

where $h_{B M j t}$ denotes channel coefficient of $j^{\text {th }}$ antenna and time slot $t$ from BS to $\mathrm{MS}_{\mathrm{k}}, h_{R M t}$ indicates channel coefficient from RS to $\mathrm{MS}_{\mathrm{k}}$ at time slot $t$ of second transmission from RS to $\mathrm{MS}_{\mathrm{k}}, r_{b i, j}$ represents the received signal at $i^{\text {th }}$ time slot of $j^{\text {th }}$ transmission, the normalization factor $\mu$ is given as

$$
\mu=\frac{1}{\left(\begin{array}{c}
\left|h_{B M 11}\right|^{2}+\left|h_{B M 21}\right|^{2} \\
+\left|\left(h_{B M 12}+h_{R M 1}\right)\right|^{2}+\left|\left(h_{B M 22}+h_{R M 2}\right)\right|^{2}
\end{array}\right)}
$$

In order to decode EL, we need to get the residual matrix $\Delta \boldsymbol{R}_{b}$ by subtracting the both received signals with $\mathbf{C}_{\mathbf{1}}$.

$$
\Delta \mathbf{R}_{b}=\left[\begin{array}{c}
\boldsymbol{r}_{b, 1}-\boldsymbol{h}_{B M} \widehat{\mathbf{C}_{\mathbf{1}}} \\
\boldsymbol{r}_{b, 2}-\left(\boldsymbol{h}_{B M}+\boldsymbol{h}_{R M}\right) \widehat{\mathbf{C}}_{\mathbf{1}}
\end{array}\right]
$$

where $\Delta r_{b i, j}$ represents the residual received signal at $i^{\text {th }}$ time slot of $j^{\text {th }}$ transmission, the normalization factor $\mu$ is given as

The estimation of EL is determined by the same above decoding technique.

Scheme II. The received signal at RS and $\mathrm{MS}_{\mathrm{k}}$ are similar to Eq. (8), (9). In the second time slot, only RS sends signal to $\mathrm{MS}_{\mathrm{k}}$ after decoding, re-encoding and forwarding.

$$
\boldsymbol{r}_{b, 2}=\sqrt{\frac{P_{r}}{2}} \boldsymbol{h}_{R M} \widehat{\mathbf{c}_{\mathbf{1}}}+\boldsymbol{n}_{R M}
$$

Eq. (11) and (12) are used to decode BL. we subtract out the term with $\mathbf{C}_{\mathbf{1}}$ in $\boldsymbol{r}_{b}$ of (9) for estimating EL.

Scheme III. The BS only broadcasts BL to RS and MSs at the first time slot. The signal matrix at the RS and the signal vector received at $\mathrm{MS}_{\mathrm{k}}$ in the first time slot are given as, respectively

$$
\begin{aligned}
& \boldsymbol{R}_{r}=\sqrt{\frac{P_{b}}{2}} \boldsymbol{H}_{B R} \mathbf{C}_{\mathbf{1}}+\boldsymbol{N}_{B R} \\
& \boldsymbol{r}_{b, 1}=\sqrt{\frac{P_{b}}{2}} \boldsymbol{h}_{B M} \mathbf{C}_{\mathbf{1}}+\boldsymbol{n}_{B M}
\end{aligned}
$$


The received signal at $\mathrm{MS}_{\mathrm{k}}$ in the second time slot is similar to Eq. (10). Both layers can be decoded by using same Alamouti decoding standard as scheme I.

\section{III.PERformance EVAluAtion}

\section{A. Bit Error Rate Performance}

In this section, we investigate the effectiveness of the various transmission schemes by using BER performance simulation. The analysis of each scheme is provided to give the optional choice for each application according the trade off of different importance of scalable layers. We also make a comparison with non-cooperative transmission case in which $\mathrm{MS}_{\mathrm{k}}$ only receives one signal consisting of the BL and EL.

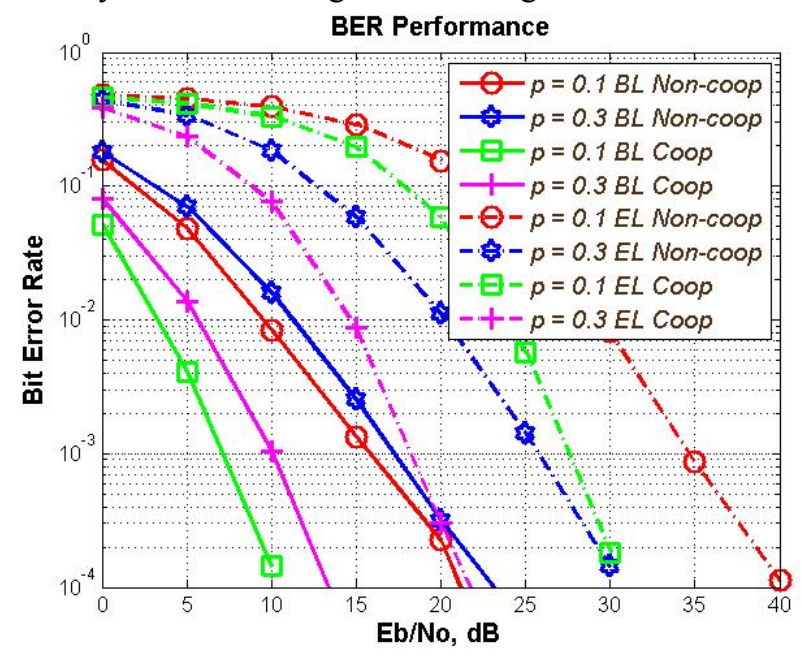

Figure 2. The BER performance of Scheme I.

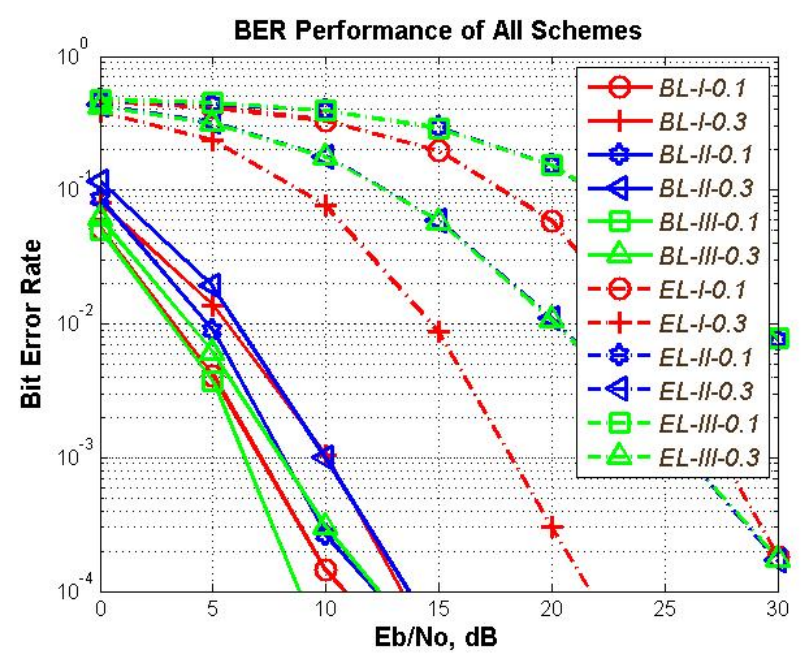

Figure 3. The BER performance of all schemes with the various amplitude ratio value, $\rho$. The elements in the legend are ordered as (layer name, scheme order, $\rho$ ).

The BER performance with QPSK modulation of both cooperative and non-cooperative transmissions for Scheme I is shown in Figure 2. The solid lines and dash-dot lines represent the BER of BL and ELs, respectively. The results show that MSs can decode both layers and enjoy the full quality of scalable video. The solid lines with square maker and plus maker are the BER of BL of Scheme I with amplify ratio values $\rho=0.1$ and $\rho=0.3$, respectively. Scheme I significantly provides $12 \mathrm{~dB}$ better protection for base layer at BER of $10^{-4}$. The BER of EL of Scheme I also give $7 \mathrm{~dB}$ better performance than non-cooperative. Different values of amplify ratio give different BER performance.

The Figure 3 shows the BER performance of all schemes at $\rho=0.1$ and $\rho=0.3$. BER behaviour of BL is always better than EL in all cases. It means that the protection of BL is better than EL. In case of amplitude ratio $\rho=0.1$, Schemes I, II and III have nearly same BER behaviour of base layer, but EL of Scheme I is observed for $7 \mathrm{~dB}$ better performance at BER of $10^{-2}$ since $\mathrm{MS}_{\mathrm{k}}$ gets two information signals of ELs. On the other hand of $\rho=0.3$, the third scheme exhibits the best BER behaviour of BL and experiences same performance of layer 2 with Scheme II. The scheme II gives the best performance of energy efficiency because BS only broadcasts signal at the first time slot. We increase the value of $\rho$ to enhance the importance of EL. The performance of ELs with $\rho=0.3$ is much better than $\rho=0.1$. Therefore, the trade off between BL and ELs could be operated by controlling the $\rho$ value.

\section{B. Scalable Video Performance}

For the comparison of video quality, the Foreman sequence is partitioned into two layers with same size and SNR scalability by using JSVM 9.19 reference software [13]. The sequence contains 150 frames with the frame rate of 15 frames/s. The group of picture (GOP) is 16 , and the resolution of both layers is $352 \times 288$. We use the peak signal to noise ratio in order to evaluate the scalable video performance given in Figure 4.

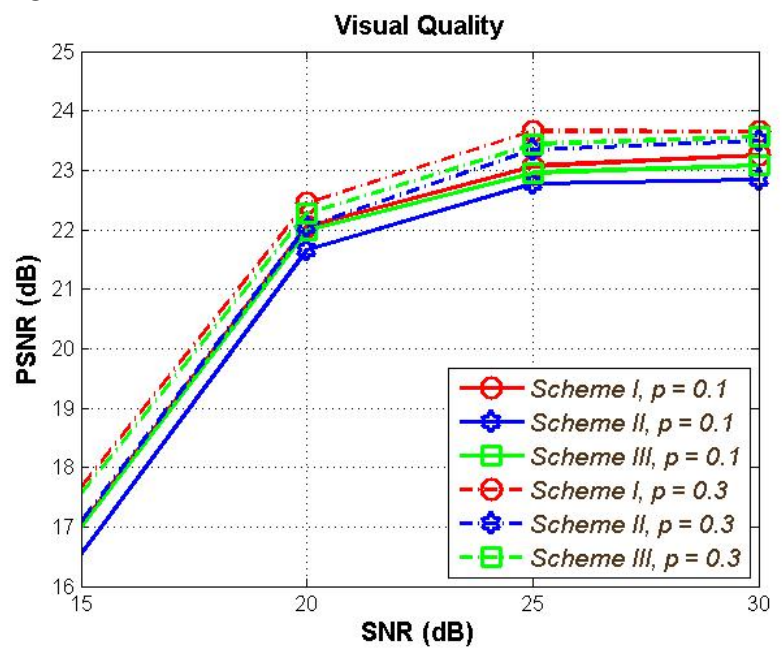

Figure 4. Video quality PSNR versus channel SNR of the Foreman sequence.

The results show that the PSNR of all schemes with various $\rho$ values is quite different. We don't consider the channels with SNR lower than $15 \mathrm{~dB}$ since there is no meaning of the PSNR below $20 \mathrm{~dB}$. Otherwise, for the channels with SNR range from $20-30 \mathrm{~dB}$, the scheme I obtains better video quality 
of average $0.3 \mathrm{~dB}$ gain than others. This is reasonable since the BER performance of the BL keeps unchanged at around $10^{-5}$ while the EL behaviour is getting better. Therefore, the receivers can decode successfully both layers with more refinement information and get the SNR scalability. The PSNR of scheme II is the lowest although having the same BER behaviour of the EL because the scheme III gets the better BER performance of the BL. Secondly, the video quality with $\rho=0.3$ gives average $0.5 \mathrm{~dB}$ gain better performance than $\rho=0.1$ from the range $20-30 \mathrm{~dB}$ since the MSs get more refinement information while getting same base layer information.

\section{IV.CONCLUSIONS}

In this paper, we investigate the comparison of transmission schemes for scalable video broadcast system over MIMO relay networks in order to provide higher protection of base layer. The simulation results show that all cooperative schemes could be used for conveying scalable video. MSs with cooperative communication could be guaranteed the basic video quality. Scheme I gives average $0.3 \mathrm{~dB}$ gain better than others because the refinement information carried in embedded STC provides the improvement of visual quality and helps users enjoy the full quality of supported SVC bit stream. The scheme II is average $0.3 \mathrm{~dB}$ gain worse than scheme III but providing more energy-efficiency. The uniform PSNR gaps are generally around $0.5 \mathrm{~dB}$ between various schemes with different amplitude ratio values. Based on the need of users and provided service, the trade off between the protection of base and enhancement layers are considered by adjusting the power dividing factor to select the suitable scheme.

For future work, the scalable video broadcast system could be significantly improved by considering the integration of error concealment, advanced channel coding and network coding. The combination and optimized design for practical video broadcast system are the attractive research and challenging topic.

\section{ACKNOWLEDGMENT}

This research was funded by the MSIP (Ministry of Science, ICT \& Future Planning), Korea in the ICT R\&D Program 2013.

\section{REFERENCES}

[1] Arogyaswami J. Paulraj, Rohit U. Nabar, and Helmut Bölcskei, "An overview of MIMO communications-A key to gigabit wireless," Proceedings of the IEEE, vol. 92, no. 2, pp. 198-218, Feb. 2004.

[2] Heiko Schwarz, Detlev Marpe, and Thomas Wiegand, "Overview of the scalable video coding extension of the H.264/AVC standard," IEEE Trans. on Circuits and Systems for Video Tech., vol. 17, no. 9, pp. 1103-1120, Sep. 2007.

[3] T. Tillo, E. Baccaglini, and G. Olmo, "Unequal protection of video data according to slice relevance," IEEE Trans. Image Process., vol. 20, no. 6, pp. 1572-1582, June 2011.

[4] Y.-R. Tsai and Y.-C. Chen, "Multilevel coupling modulation for multiresolution multimedia broadcast/multicast service in OFDM systems," IEEE Trans. Commun., vol. 59, no. 1, pp. 141-150, Jan. 2011.
[5] A. Sendonaris, E. Erkip, and B. Aazhang, "User cooperation diversity part I: System description," IEEE Trans. Commun., vol. 51, no. 11, pp. 1927-1938, Nov. 2003.

[6] A. Nosratinia, T. Hunter, and A. Hedayat, "Cooperative communication in wireless networks," IEEE Commun. Mag., vol. 42, no. 10 , pp. $74-80$, Oct. 2004.

[7] T. M. Cover and A. A. E. Gamal, "Capacity theorems for the relay channel," IEEE Trans. Inform. Theory, vol. 25, no. 5, pp. 572-584, Sep. 1979.

[8] G. Kramer, M. Gastpar, and P. Gupta, "Cooperative strategies and capacity theorems for relay networks," IEEE Trans. Inform. Theory, vol. 51, no. 9, pp. 3037-3063, Sep. 2005.

[9] G. Kramer, M. Gastpar, and P. Gupta, "Fading relay channels: performance limits and space-time signal design," IEEE J. Selected Areas Commun., vol. 22, no. 6, pp. 1099-1109, Aug. 2004.

[10] S. M. Alamouti, "A simple transmit diversity technique for wireless communication," IEEE J. Selected Areas Commun., vol. 16, no. 8, pp. 1451-1458, Oct. 1998.

[11] C.-H. Kuo and C.-C. J. Kuo, "An embedded space-time coding scheme for broadcasting," IEEE Trans. Broadcasting, vol. 53, no. 1, pp. 48-58, Mar. 2007.

[12] C.-H. Kuo, C.-M. Wang, and J.-L. Lin, "Cooperative wireless broadcast for scalable video coding," IEEE Trans. on Circuits and Systems for Video Techn., vol. 21, no. 6, pp. 816-824, June 2011.

[13] SVC Reference Software. [Online]. Available: http://www.hhi.fraunhof er.de/de/kompetenzfelder/image-processing/research-groups/imagevideo-coding/svc-extension-of-h264avc/jsvm-reference-software.html

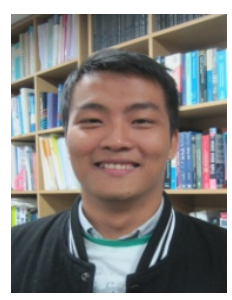

Phuc Chau received the B.S. degree from Hochiminh University of Science, Vietnam in 2010. He is currently a M.S. candidate in the Department of Electronic, Electrical and Computer Engineering, College of Information and Communication Engineering, Sungkyunkwan University, Rep. of Korea. His research interests include video signal processing and transmission over next generation Internet and wireless/mobile networks, $5 \mathrm{G}$ communication systems, and multimedia network control/protocol issues.

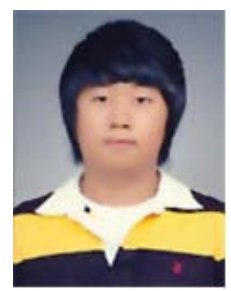

Yong-woo Lee received the B.S. degree from Sungkyunkwan University in 2013. He is currently a M.S. candidate in the Department of Electronic, Electrical and Computer Engineering, College of Information and Communication Engineering, Sungkyunkwan University, Rep. of Korea. His research interests include video signal processing and transmission over next generation Internet and wireless/mobile networks, $5 \mathrm{G}$ communication systems, and medical image processing.

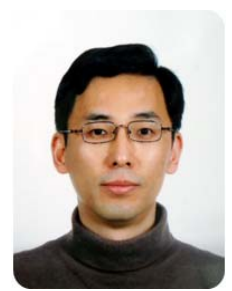

Jitae Shin received the B.S. degree from Seoul National University in 1986, the M.S. in Korea Advanced Institute of Science and Technology (KAIST) in 1988. After 8 years working in Korea Electric Power Corp. (KOPEC) and Korea Atomic Energy Research Institute (KAERI), he returned to study and received the M.S. and Ph.D. degrees in Electrical Engineering from University of Southern California, Los Angeles, U.S.A. in 1998 and 2001, respectively. $\mathrm{He}$ is a Professor in College of Information and Communication Engineering of Sungkyunkwan University, Suwon, Korea. His research interest includes video signal processing and transmission over next generation Internet and wireless/mobile networks, 5G communication systems, and multimedia network control/protocol issues. 